\title{
La formation en gestion et les pratiques d'adaptation de l'enseignement dans le cadre de la formation transfrontalière
}

\author{
Najat GLABI
}

L'enseignement dans les programmes de l'université engagée dans une formation transfrontalière est assuré par les professeurs de cette même université, selon les mêmes modalités que sur son propre campus, mais à des étudiants étrangers qui restent eux, dans leur pays d'origine et peuvent dans certains cas, recevoir la formation dans les locaux d'une institution partenaire dans le pays d'accueil. Il s'agit de l'enseignement transfrontalier. Les professeurs qui offrent un enseignement transfrontalier ont pour mandat de donner les mêmes cours qu'ils offrent sur le campus de leur université d'origine, avec le maintien du même objectif; ce qui revient à dire que les méthodes d'évaluation des acquis

\section{L'enseignement transfrontalier : une universitaire}

Les formations transfrontalières répondent à une demande solvable exprimée à l'échelle internationale et concrétisent un choix politique des dirigeants des universités de renforcer leur processus d'internationalisation. Elles permettent aux universités d'offrir leurs formations et de délivrer leurs diplômes en dehors de leurs campus et même en dehors des frontières de leurs pays d'origine. Les formations transfrontalières sont à l'origine de l'enseignement transfrontalier qui est une pratique récente dans les universités et une réalité peu documentée. Les professeurs qui offrent cet enseignement se constituent, élaborent et testent et ce, au fil des expériences sont les mêmes et garantissent le même niveau de compétence. Or, offrir un cours dans un contexte international met le professeur dans une situation inhabituelle et l'incite à amorcer une réflexion et à agir pour s'adapter au changement du contexte dans lequel il enseigne. L'objectif de notre étude est de décrire le travail d'autoévaluation et d'adaptation effectué par les professeurs et d'identifier des stratégies d'enseignement nouvelles, pensées et appliquées à une réalité différente qui est l'enseignement transfrontalier mais ayant néanmoins, les mêmes exigences de qualité que l'enseignement sur le campus de l'université.

\section{nouvelle dimension du travail enseignant} vécues, des pratiques et des stratégies d'enseignement qui leur permettent de maintenir l'efficacité de leur enseignement en dépit du changement de contexte.

Le modèle théorique que nous avons utilisé pour étudier les adaptations effectuées par les professeurs sur leurs stratégies pédagogiques lorsqu'ils sont dans un contexte transfrontalier est le modèle pour la recherche en pédagogie élaboré par Gauthier et al (1997). Ce modèle représente une conceptualisation de ce qui se passe en classe à partir des deux fonctions principales du professeur, à savoir, la gestion de la matière et la gestion de la classe. 


\section{La méthodologie}

Pour connaître les adaptations introduites par les professeurs dans le cadre de leur enseignement transfrontalier, nous avons réalisé une étude de cas multiple basée sur l'expérience de douze professeurs de l'Université du Québec à Chicoutimi. Les professeurs qui offrent leur enseignement dans le cadre des programmes transfrontaliers en administration des affaires représentent les cas de notre étude. Le cours offert tel que défini par les protocoles d'ententes qui régissent les programmes franchisés de l'Université du Québec à Chicoutimi nous servira d'unité d'analyse.

\section{La composition de l'échantillon par le recrutement des cas}

Notre échantillon est composé de douze professeurs $(\mathrm{N}=12)$ qui participent aux formations transfrontalières offertes par l'Université du Québec à Chicoutimi (UQAC) dans deux pays différents, à savoir, le Maroc et le Sénégal. Les professeurs participants à notre étude sont des professeurs efficaces sur le campus de l'Université. Le critère d'efficacité est confirmé par la reconnaissance des pairs. Ainsi, l'échantillon a été composé sur la recommandation des responsables de projet par pays au sein de l'université.

\section{La collecte des données}

Par souci d'accroître la validité interne de notre étude, nous avons procédé à une collecte de données par triangulation. Ce qui signifie le recours à l'étude documentaire, aux entrevues individuelles et à l'observation en milieu naturel, à savoir en classe dans un pays autre que le Canada.
- L'étude documentaire consiste en la collecte et l'analyse du contenu des documents tels que les protocoles d'ententes qui régissent les formations transfrontalières de l'UQAC, les documents internes qui traitent de la mise en place et de l'évaluation de ces formations transfrontalières, les plans de cours et le matériel pédagogique utilisé par les professeurs dans le cadre de leur enseignement transfrontalier (Merriam 1998, Mucchielli 1991).

- Les entrevues individuelles et semi dirigées se déroulent selon le canevas d'entretien avec les professeurs participants sur une durée de quatre heures réalisées en deux temps. Les entrevues avec les professeurs ont pour objectif d'expliciter les adaptations des enseignements dans un contexte transfrontalier, d'amorcer une réflexion sur ces adaptations et d'effectuer un retour sur cette réflexion au terme de l'étude pour valider les conclusions de l'étude (Bourassa et al. 1999, Bassey 1999).

- L'observation non participante et répétée en classe pendant deux séances de cours pour chaque professeur en contexte transfrontalier, et ce, dans deux pays, le Maroc et le Sénégal. L'objectif de l'observation est de décrire les stratégies pédagogiques adaptées par lesquelles les professeurs maintiennent l'efficacité de leur enseignement. Elle permet de déterminer s'il existe un écart entre le discours professionnel des professeurs (la théorie professée) et leurs pratiques professionnelles (théorie pratiquée) (Schön 1983). 


\section{Les résultats de l'étude : L'adaptation des enseignements dans les formations transfrontalières}

L'enseignement inclut une phase d'interactivité qui rend le maintien de son efficacité tributaire d'éléments subjectifs et incontrôlables comme l'affectivité, la culture et l'éthique. L'enseignement ne peut donc, être reproduit à l'identique d'un lieu à l'autre et encore moins réaliser les mêmes effets d'efficacité d'un pays à l'autre (AbdellahPretceille 2004, Murphy 2007).

\subsection{S'adapter...à quoi?}

Dans la pratique de l'enseignement transfrontalier, les faits mettent en évidence des adaptations introduites par les professeurs pour gérer des contraintes d'ordre culturel, académique et organisationnel. Ces contraintes sont propres à l'enseignement transfrontalier et résultent essentiellement du changement de contexte dans lequel le professeur offre son enseignement.

\section{S'adapter à un contexte culturel différent}

En classe, les professeurs adoptent des comportements en référence à leur propre environnement culturel alors que les étudiants se réfèrent à un environnement culturel différent. Le risque de choc culturel dans la classe pour l'étudiant incite les professeurs à introduire des changements de comportement et d'attitude pour maintenir l'efficacité de leur enseignement (Pyvis et Chapman 2005). L'adaptation des stratégies pédagogiques en tenant compte des différences culturelles confère une dimension interculturelle à l'enseignement transfrontalier et conditionne son efficacité (Abdellah-Pretceille 2004, Aron 1989).

\section{S'adapter à un système académique différent}

D'un pays à l'autre, les systèmes éducatifs assurent des niveaux de connaissances différents, même si sur le plan administratif, des procédures d'équivalence ${ }^{1}$ permettent un rapprochement, le plus souvent sur la base du nombre d'années de scolarité et non sur la base du contenu de la formation. Le professeur est appelé à offrir un cours qui suppose des prérequis sans qu'il puisse s'assurer si l'étudiant étranger les possède ou pas. Le passage d'un système éducatif à un autre incite les professeurs à adapter leur enseignement de manière à maintenir la cohérence du curriculum (Schmidt et Prawat 2006). Le changement de système éducatif soulève la question de la maîtrise de la langue d'enseignement. En effet, l'enseignement primaire et secondaire est souvent offert dans la langue officielle du pays d'accueil qui est différente de la langue d'enseignement dans l'université d'origine. Ce passage de l'apprentissage d'une langue à l'autre réduit les capacités d'interaction et d'apprentissage des étudiants des formations transfrontalières et incite les professeurs à s'adapter à cette contrainte pour maintenir l'efficacité de leur enseignement (Debowski 2005). L'enseignement offert dans le cadre des formations transfrontalières a pour objectif de développer chez les étudiants internationaux des compétences compatibles avec les réalités socioéconomiques du pays d'accueil. Dans leur quête du maintien de l'efficacité de leur enseignement transfrontalier, les professeurs tiennent compte de cet objectif lors de l'élaboration des cours qu'ils offrent à l'international (Murphy 2007).

\section{S'adapter à un contexte organisationnel différent}

D'une université à l'autre, l'organisation de la scolarité change de manière notable, de même avec l'établissement étranger qui accueille les programmes franchisés, cette organisation est différente de celle adoptée dans l'université 
dont les programmes sont issus. Il s'agit là de tous les aspects organisationnels qui vont du règlement intérieur de l'institution jusqu'à la planification des cours. L'organisation des interventions des professeurs est préalablement déterminée dans le protocole d'entente qui lie les deux institutions partenaires. Cette composante de l'accord influence les capacités des professeurs ainsi que celles des étudiants à atteindre les objectifs d'apprentissage fixés. Elle touche plusieurs aspects du travail du professeur comme la gestion du temps ou les règles qui régissent les rapports professeurétudiant. Les principales contraintes identifiées par les travaux de recherche concernent la planification intensive des interventions des professeurs à l'international (Dunn et Wallace 2007 ; Debowski 2005), les rapports que le professeur entretien avec le personnel de l'institution partenaire dans le pays d'accueil (Leask 2001; Debowski 2005) ainsi que l'encadrement offert par l'université d'origine qui doit être plus soutenu que celui offert aux professeurs lors de leur enseignement sur le campus de l'université ( Pyvis et Chapman 2005).

\subsection{Adapter...quoi ?}

Lors d'un enseignement transfrontalier, les professeurs modifient leur mode de gestion de la matière qu'ils enseignent pour le rendre plus adapté au contexte culturel et au système académique du pays d'accueil d'une part et au contexte organisationnel de l'institution partenaire d'une autre part.

- La dimension interculturelle de l'enseignement

La gestion $d u$ temps. Les professeurs interviewés ont constaté une différence dans le rapport au temps chez les étudiants marocains et sénégalais. Cette différence se manifeste chez les étudiants par un manque de ponctualité et le non respect des échéances de la remise des travaux.
L'exercice de l'autorité. Les professeurs font état d'un rapport à l'autorité chez les étudiants marocains et sénégalais différent de celui des étudiants au Québec. Les professeurs interrogés considèrent que leur crédibilité dans la classe est remise en question par les étudiants et sont incités à structurer différemment le contenu de leur matière pour regagner cette crédibilité lors de leur enseignement transfrontalier. Les professeurs sont interpelés par le manque de motivation des étudiants et le caractère obligatoire des apprentissages.

Les rapports au genre. Les rapports au genre sont un construit social et sont influencés par le changement de contexte social. Les professeurs en tiennent compte et adaptent leur mode de gestion des travaux d'équipe lorsqu'elles sont mixtes ainsi que le contenu de leurs enseignements pour ménager les susceptibilités culturelles chez les étudiants marocains et sénégalais. Les professeurs masculins tiennent compte de ces susceptibilités dans la gestion de l'apprentissage de leurs étudiantes du genre féminin, tandis que la professeure qui fait partie de l'échantillon a reconnu tenir compte de cette susceptibilité dans la gestion de l'apprentissage de ses étudiants du genre masculins.

La gestion des équipes. Tous les professeurs interrogés recourent aux travaux d'équipes comme mode d'apprentissage. Adopter cette méthode dans le contexte transfrontalier incite les professeurs à tenir compte du sentiment d'appartenance au groupe chez les étudiants marocains et sénégalais, particulièrement lors de la répartition des tâches entre les différents membres d'un groupe et lors de l'évaluation des travaux rendus par les équipes.

Le recours à l'humour. Les professeurs citent l'humour comme un moyen de présenter les connaissances en dépassant les différences culturelles avec leurs étudiants au Maroc et au 
Sénégal. Ils ont recours à l'humour plus fréquemment dans un contexte transfrontalier que lors de leur enseignement sur le campus et le considèrent comme un vecteur de la communication interculturelle.

Les savoirs disciplinaires. Les professeurs soulignent le caractère universel des savoirs disciplinaires à haut niveau de technicité et reconnaissent le mettre en avant dans un contexte transfrontalier plus fréquemment que dans les enseignements sur le campus.

Le respect. La notion de respect se définit différemment d'un contexte culturel à un autre. Les professeurs interrogés déclarent avoir relevé ces différences et considèrent comme indispensable la définition d'un sens commun du respect avec leurs étudiants marocains et sénégalais et ce, au début de chaque cours. Il s'agit d'un contrat moral qu'ils présentent à leurs étudiants comme préalable à toute activité d'éducation interculturelle.

Les différences culturelles. Les professeurs reconnaissent capitaliser sur les différences culturelles avec les étudiants marocains et sénégalais pour stimuler l'intérêt des étudiants et renforcer leurs capacités d'apprentissage.

- Offrir un enseignement dans une langue seconde

Le degré de difficulté du vocabulaire utilisé. Les formations transfrontalières offertes par l'Université du Québec à Chicoutimi au Maroc et au Sénégal reconnaissent la maîtrise de la langue d'enseignement, en l'occurrence le français comme une exigence d'admission aux programmes. Les professeurs interrogés sont avertis de cette condition avant leur déplacement dans ces deux pays. Ils relèvent pourtant des lacunes dans la maîtrise de la langue qui les incitent à adapter le vocabulaire qu'ils utilisent lors de leur enseignement pour favoriser l'apprentissage des étudiants marocains et sénégalais.

L'usage de la langue française à l'oral. Les professeurs considèrent que le niveau de maîtrise de la langue française chez les étudiants marocains et sénégalais est suffisante à l'oral et n'affectent ni la qualité ni la fréquence des interactions en classe. Ils maintiennent ainsi le même niveau de leur vocabulaire lors de leur enseignement mais reconnaissent éviter les expressions spécifiques aux régions pour adopter un vocabulaire plus « international ».

L'usage de la langue française à l'écrit. Les professeurs interrogés ont relevé une faible maîtrise de la langue française à l'écrit à travers les évaluations des étudiants marocains et sénégalais. Le caractère presque généralisé de cette problématique les a incités à ne pas tenir compte des fautes de français lors de l'évaluation de ces étudiants et à mettre l'accent sur les savoirs disciplinaires pour déterminer la note finale.

- Tenir compte du niveau des connaissances préalables chez les étudiants

Interdisciplinarité et esprit de synthèse. Dans le cadre de leurs cours sur le campus, les professeurs ont déclaré faire recours à l'interdisciplinarité et à l'esprit de synthèse pour développer les compétences des étudiants. Cette stratégie est peu utilisée dans le cadre de l'enseignement transfrontalier au Maroc et au Sénégal du fait que les étudiants acquièrent les connaissances dans le cadre de disciplines cloisonnées et ont été peu incité à développer leur esprit de synthèse.

La méthodologie. Les professeurs ont remarqué une tendance chez les étudiants marocains et sénégalais à recourir à la mémorisation pour acquérir des connaissances. Les professeurs mettent l'accent sur les travaux pratiques et les applications pendant le 
cours pour inciter les étudiants à développer leur esprit critique et leur créativité et limiter ainsi le recours systématique à la mémorisation.

Les savoirs disciplinaires. Les professeurs ne connaissent pas le niveau des pré-requis des étudiants lors de la phase de préparation du cours. C'est une fois sur place, au début de chaque cours, qu'ils évaluent les connaissances préalables des étudiants par le questionnement. Les professeurs les plus expérimentés consacrent un temps déterminé pour cette phase dans le cadre de leur planification du cours ainsi qu'un temps consacré au retour sur l'explication des concepts fondamentaux pour combler les lacunes des étudiants.

- Adapter le contenu des cours aux besoins du pays d'accueil

La stratification de la société d'accueil. Les formations transfrontalières de l'UQAC sont offertes dans des institutions partenaires privées. Les étudiants inscrits dans ces formations s'acquittent de frais de scolarité aux montants très élevés en comparaison avec le niveau de vie du pays d'accueil (les frais annuel représentent le double du salaire minimum marocain- SMIG). La mobilité entre les classes sociales est limitée dans certains pays comme le Maroc et l'appartenance à une classe sociale offre des privilèges en matière d'emploi et de rémunération sans référence aux compétences. Cette logique met en échec la logique de méritocratie sur laquelle les professeurs s'appuient pour stimuler la motivation des étudiants pour l'apprentissage.

L'environnement économique. Les formations transfrontalières sont offertes dans des pays au niveau de développement différent du pays d'origine (Canada). Le tissu économique est souvent composé essentiellement de petites et moyennes entreprises et le mode de gestion traditionnel est prédominant. Les professeurs de l'UQAC tiennent compte de cette réalité et y puisent des exemples et des cas d'entreprises locales pour stimuler les apprentissages des étudiants.

Les différences entre le premier cycle et le deuxième cycle. Les professeurs interrogés ont relevé une nette distinction dans les attentes des étudiants en matière d'apprentissage et d'approche pédagogique selon qu'ils sont inscrits à des programmes de premier cycle ou de deuxième cycle. La clientèle du premier cycle est composée essentiellement de jeunes étudiants qui n'ont pas encore intégré le marché de l'emploi et dont la motivation pour les apprentissage relève plus du sens du devoir puisque le financement de leur formation est souvent pris en charge par leurs parents; tandis que la clientèle du deuxième cycle est composé de jeunes cadres en exercices dont la formation est financée par l'entreprise dans laquelle ils exercent ou à partir de leurs propres revenus pour accélérer une promotion professionnelle. Cette clientèle a une vision très utilitaire de la formation et cherche à développer des compétences qui lui permettront d'être plus efficace en entreprise le plus rapidement possible. Les professeurs adaptent le contenu de leur cours et leurs stratégies pédagogiques de manière à capitaliser sur ces attentes et favoriser l'acquisition des connaissances.

- Tenir compte de la planification intensive des cours

Le maintien des objectifs d'apprentissage. La planification des cours offerts dans les formations transfrontalières est différente de celle adoptée pour les mêmes cours sur le campus de l'université d'origine. Les professeurs déclarent manquer de temps pour offrir le même contenu de cours que sur le campus. Ils maintiennent les mêmes objectifs de cours mais en citant moins d'exemples et se concentrant sur les éléments fondamentaux du cours. 
Les délais d'apprentissage. Les professeurs interrogés ont relevé une inadéquation entre le temps alloué aux cours dans les formations transfrontalières et la capacité d'assimilation des étudiants. Ils considèrent que la planification intensive des cours ne respecte pas les délais d'apprentissage nécessaire à l'assimilation des connaissances prévues dans le cadre de leurs cours. Des professeurs essaient d'y remédier en communiquant le support de cours des semaines avant leur arrivée sur place pour permette aux étudiants de se familiariser avec le contenu du cours et de prendre ainsi de l'avance. D'autres professeurs fixent les dates de remise des travaux qui servent de base à l'évaluation des étudiants des semaines après la fin de leur cours pour permettre aux étudiants de disposer de plus de temps pour assimiler le contenu du cours.

L'épuisement professionnel. La planification intensive combinée à l'éloignement géographique des pays d'accueil exposent les professeurs de l'UQAC qui participent aux formations transfrontalières au risque d'épuisement professionnel. Les cours à l'international sont offerts pendant les périodes de relâche alors que les professeurs ont offert des cours sur le campus pendant toute une session, ce qui les prive de périodes de repos nécessaires à la récupération. Les professeurs les plus expérimentés reconnaissent avoir développé, au fil de leurs séjours à l'étranger, des stratégies pour gérer cet épuisement et neutraliser son effet sur l'efficacité de leur enseignement.

- Entretenir des rapports avec l'institution partenaire

La gestion de la scolarité. Les formations transfrontalières sont offertes en partenariat avec des écoles supérieures de gestion du pays d'accueil. La notion d'école de gestion suppose une gestion de la scolarité différente de celle des universités en général et de
l'UQAC en particulier. Les professeurs ont souligné cette différence et ont déclaré se conformer à certains règlements de l'institution partenaire même s'ils n'existent pas à l'UQAC, comme le contrôle des présences des étudiants en salle de cours, la remise d'un examen de rattrapage et l'exécution de sanctions disciplinaires.

Les conditions de travail des professeurs. Les professeurs ont déclaré faire face à des contraintes matérielles lorsqu'ils enseignent dans un contexte transfrontalier. Le matériel informatique est souvent défectueux ou inexistant, ce qui rend difficile le recours aux projections comme support visuel. La connexion internet est de mauvaise qualité, ce qui empêche les professeurs de planifier des activités de recherche en ligne. L'effectif de certains groupes d'étudiants atteint la soixantaine, ce qui rend difficile toute interaction en classe. Certains professeurs ont également déclaré avoir offert des cours dans des salles peu spacieuses et peu ou pas équipée pour accueillir les étudiants.

La collaboration avec les professeurs locaux. Les formations transfrontalières connaissent deux types de programmes. Des programmes offerts dans leur totalité par l'UQAC et des programmes offerts en double diplomation. Ces derniers reposent sur des mécanismes d'équivalences (reconnaissances des acquis) pour reconnaître à l'établissement partenaire le droit d'offrir et de gérer une partie de la formation donnant accès au diplôme de l'UQAC. Dans le cadre des programmes offerts par équivalence, un cours d'un volume horaire total de 45 heures de cours est offert en partie par un professeur de l'UQAC à raison de 30 heures de cours, et complété par un professeur de l'établissement partenaire à raison de 15 heures de cours. Les professeurs de l'UQAC interrogés ont reconnus ne pas avoir de contacts avec les professeurs locaux et qu'il y a un manque de coordination par rapport aux contenus du cours et aux modes 
d'évaluation. Ils considèrent que cette répartition leur fait perdre le contrôle sur les cours qu'ils offrent dans les institutions partenaires ainsi que sur la validation des acquis des étudiants.

- Bénéficier de l'encadrement de l'université d'origine

La communication interne. Les mécanismes de communication interne mis en place sur le campus de l'UQAC se révèlent insuffisants lorsqu'il s'agit de l'enseignement transfrontalier. Les professeurs interrogés ont mentionné un manque d'informations qui accentue leurs incertitudes lorsqu'ils enseignent à l'international. Il s'agit principalement d'informations d'ordre institutionnel et que les professeurs ne peuvent obtenir à titre individuel. Parmi ces informations, ils ont cité la composition des groupes d'étudiants (effectif et scolarité antérieure), les responsabilités du professeur, celles de l'institution partenaire, désigner un interlocuteur aux professeurs sur place. Certains professeurs ont souligné le besoin d'un suivi pédagogique exercé par l'université sur les cours offerts par ses professeurs dans les formations transfrontalières comme un retour sur l'action pour l'évaluation et le réajustement.

\section{Conclusion}

L'expérience des professeurs de l'UQAC nous a permis de savoir à quoi sont appelés à s'adapter les professeurs dans un contexte transfrontalier. Nous avons ainsi identifié les contraintes auxquelles s'adapter à savoir, les contraintes culturelles, académiques et organisationnelles. Nous avons également identifié les stratégies qui sont les plus souvent adaptées par les professeurs pour maintenir l'efficacité de leur enseignement. Cette
Le soutien logistique. L'éloignement géographique et le déplacement des professeurs nécessitent une gestion logistique propre aux formations transfrontalières. Les professeurs interrogés ont fait état de dysfonctionnements à ce niveau qui se traduit par des situations embarrassantes et irritantes dans le pays d'accueil. Les problèmes de logistiques sont d'autant plus éprouvants pour les professeurs qu'ils ont lieu dans un contexte qui leur est étranger et dont ils ne maîtrisent pas les codes. Les professeurs interrogés ont mentionné des problèmes de logement (hôtels peu confortables ou des réservations annulées), des problèmes de transport ou de restauration dans le pays d'accueil.

Le soutien administratif. $\mathrm{La}$ gestion administrative des formations transfrontalières a lieu conjointement entre 1'UQAC et l'institution partenaire. Les défaillances de la coordination entre les deux partenaires se répercutent sur le travail enseignant des professeurs dans le pays d'accueil. Des professeurs ont mentionné la gestion des supports de cours (qui ne sont pas prêts au début du cours), la gestion de l'évaluation des étudiants (la validation des cours a lieu sans l'implication des professeurs), la gestion de l'admission des étudiants et de la reconnaissance des acquis (les admissions parallèles gérées de manière unilatérale par l'institution partenaire).

première analyse du discours professionnel des professeurs sera suivie d'une analyse plus approfondie dont l'objectif sera d'identifier les nouvelles stratégies pédagogiques propres à l'enseignement transfrontalier pour répondre à la question «Comment adapter les stratégies pédagogiques pour assurer un même niveau de qualité des enseignements en contexte transfrontalier? ». 


\section{Notes et références}

${ }^{1}$ Procédure d'équivalence établie par le ministère de l'Éducation du Québec.

\section{Bibliographie}

ABDELLAH-PRETCEILLE, M. (2004). L'éducation interculturelle, Paris: Presses Universitaires de France.

ARON, H. (1989). Une dysfonction majeure : l'enseignement en coopération, Paris, L'Harmattan.

BASSEY, M. (1999). Case study research in educational settings, Buckingham, Open University Press.

BOURASSA, B. et al. (1999). Apprendre de son expérience. Sainte-Foy, Presses de l'Université du Québec.

CASTLE, R., \& KELLY, D. (2004). "International Education: Quality Assurance and Standards in Offshore Teaching: Exemplars and Problems." Quality in Higher Education, vol.10, n 1, p. 51-57.

PYVIS, A. \& CHAPMAN, D. (2006)."Quality, Identity and Practice in Offshore University Programmes: Issues in the Internationalization of Australian Higher Education" Teaching in Higher Education, vol. 11, $\mathrm{n}^{\circ}$ 2, Apr, p. $233-245$

DEBOWSKI, S. (2005). "Across the Divide: Teaching a Transnational MBA in a Second Language." Higher Education Research and Development, vol. 24, n 3, p. 265-280.

DUNN, L., \& WALLACE, M. (2006). "Australian Academics and Transnational Teaching: An Exploratory Study of Their Preparedness and Experiences". Higher Education Research and Development, vol. 25, n 4, p. 357369.

GAUTHIER, C. et al. (1997). Pour une théorie de la pédagogie: recherches contemporaines sur le savoir des enseignants. Québec: Les Presses de l'Université Laval.

LEASK, B. (2001). "Internationalisation: Changing Contexts and their Implications for Teaching, Learning and Assessment" Australian Society for Educational Technology and Higher Education Research and Development Society of Australia. p. 389-401

MERRIAM, S. B., (1998). Qualitative research and case study applications in education San Francisco: Jossey-Bass Publishers.

MURPHY, M. (1997). "Experiences in the Internationalization of Education: Strategies to Promote Equality of Opportunity at Monterrey Tech", The International Journal of Higher Education and Educational Planning, vol.53, $\mathrm{n}^{\circ}$ 2, Feb, p.167-208

MUCHIELLI, A. (1991). Les méthodes qualitatives (1re éd.) Paris, Presses Universitaires de France.

SCHÖN, D. A. (1983). The reflective practitioner : how professionals think in action, New York, Basic Books.

SCHMIDT, W.H \& PRAWAT, R.S. (2006) "Curriculum Coherence and National Control of Education: Issue or Non-Issue?” Journal of Curriculum Studies, vol. 38, n 6, Dec, p. 641-658.

VAN DAMME, D. (2001). "Quality Issues in the Internationalisation of Higher Education", Higher Education, vol. $41, \mathrm{n}^{\circ} 4$, p. $415-441$. 
Publicité

Revue PME 